\title{
On the Variant Character and the Determination of the Relationship between Variant Character
}

\author{
Jian-Yu LIU \\ School of Chinese Literature, Bohai University, 19 Keji Road, Songshan District, \\ Jinzhou, Liaoning, P.R.C. \\ prcmap@163.com
}

\begin{abstract}
Keywords: Variant Character, Characters with Alternative Writings (CAW), Characters with Alternative Configurations (CAC), Configuration, Function
\end{abstract}

\begin{abstract}
The variant character includes two types: Characters with Alternative Configurations and Characters with Alternative Writings, the configurational principle of the former is the same while the latter is different. The main reason of the emergence of variant character should be attributed to the relentless pursuit on the more accurate, clear and easy to record Chinese. On determination of the variant character, we must take the integrated survey on configuration and function as a fundamental principle. Firstly, investigate whether the characters in the character set to be analyzed were invented for the same word from the configuration perspective. If it isn't, then investigate whether it can be associated by homophone borrowing from the function. Finally, investigate whether the function of characters in the character set to be analyzed has differentiated during use from the functional perspective.
\end{abstract}

\section{The Definition and Classification of Variant Character}

The use of variant Chinese Character is a kind of excrescence, so the Chinese ancient scholars have noticed this kind of phenomenon very early, and carried out collating and researching for several times. After the founding of the P.R.C, with the deepening of Chinese Character collating work and officially announcing of the First Batch of Tabulated Variant Forms of Chinese Character in 1955, the term "variant" began to generally accepted and used. However, there is still a lot of debate on the definition and specific classification of Variant Chinese Character in the research and collating process. Mr. You Mingzhi lists the visible definitions of variant character as many as 17 in his doctoral papers. [1]

Mr. Li Guoying believes that the variant character refers to the different forms invented for the same word which the function doesn't differentiate and the different forms of a character caused by the writing variation. [2] The variant character includes two types: Characters with Alternative Configurations (CAC) and Characters with Alternative Writings (CAW). The CAC refers the different forms invented for the same word which the function doesn't differentiate, it is derived from the configuration and belongs to the inventing variant character. The CAW refers the different forms of a character caused by the writing variation, it is derived from writing and belongs to writing variant character. CAC has the same word function but is different in configuration attributes. The difference is mainly embodied in the four aspects of component quantity, structure relation, selected component and component orientation. CAW is the same as the word function and the configuration attributes, but the writing attributes such as the number of strokes and pen strokes are slightly different caused by the basic components of the writing variation. It includes the CAW with slight differences in strokes, the mixed-up CAW caused by close-up shaped and the transferred written character. The essential difference between $\mathrm{CAC}$ and $\mathrm{CAW}$ is that CAC comes from the configuration and belongs to invention variant while CAW comes from the writing and belongs to written variant.

Mr. Sun Jianwei believes that configurational principle of CAW is the same, but it is different in CAC. CAW contains two types: the variant character on stroke dimensions and on static structure level. The latter also can be dividing into two types: some are different in component location while the other are in the number of components. CAC mainly refers to the variant character which are dynamic component dimensions, it can be dividing into two types: some are different in the creation 
method while others are the same in creation method but different in the components.

\section{The Motive of the Variant Character}

Mr. Ma Jinglun believes that the reason for the emergence of variant character mainly because the invention of Chinese Character is the result of the collective wisdom and in such a large area, so many people use, therefore, it is inevitable to invent two or many Chinese Characters to record a same word. [4] However, multi-source of invention of Chinese Character is the superficial reason of the emergence of variant character, the main reason should be attributed to the relentless pursuit on the more accurate, clear and easy to record Chinese. With the continuous development of Chinese, when the original Chinese Character can't be accurate, clear and easy to record Chinese, people will invent or write many variant characters inevitably under the rule of express, the rule of simple and easy and the rule of dissimilarity. Many of them could be included into the ancient dictionaries and inheritance so far.

First, the rule of express requires Chinese Character to timely and accurately records the words in the language and tries to do the unity of shape, pronunciation and meaning. On the one hand, the glyph should maximize the corresponding to the pronunciation and meaning when the glyph to be formed for the same word in the language. The non-conformance glyphs will be reformed by adding (changing) the components, even the conformance glyphs are also constructed by adding (changing) the components from the other perspectives of the meaning. On the other hand, the choice of the configuration pattern will be influenced and constrained by the rule of express during the Chinese Character configuration. When the original form of the configuration doesn't meet the requirements of recording the word, new glyph will be considered to invent using the new configuration model naturally, and will be more inclined to choose the phonetic mode which is on the dominant position in general.

Second, the rule of simple and easy requires the structure of Chinese Character to be as simple and clear as possible to make the writing and the learning more convenient. When the glyph of Chinese Character is too cumbersome or the motivation is too obscure, people will take the initiative to economize some tricky configuration of the Chinese Character or replace the original structure with other similar configuration under the influence of the rule of simple and easy to make the glyph structure as simple as possible to facilitate writing and remember. It is feasibly mainly because there is a certain compatibility of the recognition of Chinese Character.

Finally, the rule of dissimilarity requires the Chinese Character has a considerable degree of distinction to facilitate the identification from the perspective of recognition. Therefore, it will be considered to change the components to achieve the purpose of enhancing the distinction degree when some Chinese Characters have the possibility of intangible confuse during the use of variation due to factors.

\section{The Principle, Processes and Methods of Determining the Variant Character}

\section{(A) The Decision Principle}

On determination of the variant character, it should take the integrated survey on configuration and function as a fundamental principle. Specifically, it is about two aspects, one is to examine whether they record the same words in the language; the other is to examine whether they have the same usage in the literature. [5]

\section{(B) The Decision Process}

The processes of determining the variant character as shown in Figure.1: 


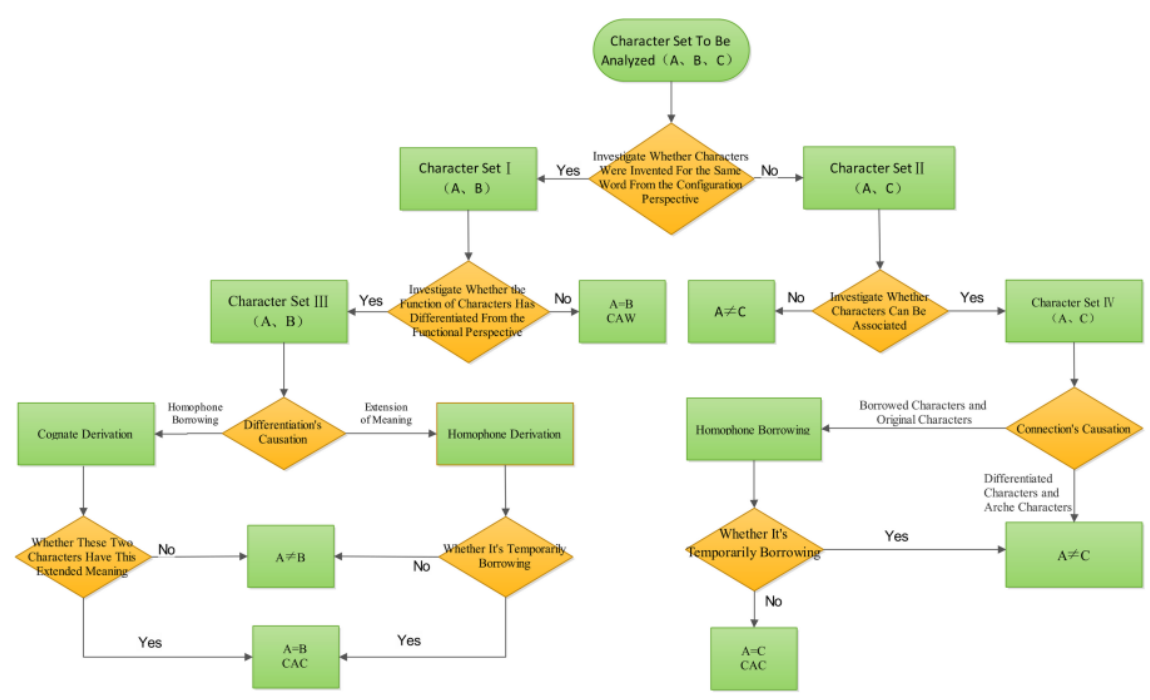

Figure. 1. The Determining Process of the Various Character.

\section{(C) The Decision Methods and Instructions}

The characters in the character set to be analyzed include those which have been invented for the proper nouns and the monomorphemic words.

(2) Investigate whether the characters in the character set to be analyzed were invented for the same word from the configuration perspective, in other words, whether the original meaning is same. The traditional identify method is to consider whether those glyphs being invented can record the same words in the practical application firstly. The result that different characters record the same word can caused by three cases: spelling variation of the character, invent the different character for the same words, the differentiation and borrowing of character, but only the first two cases can produce the variant character. Therefore, it is inappropriate both in theory and in practice to identify the variant character from this starting point because it will inevitably impose a different relationship in the nonalluvial character and take the different characters which partial coincidence of function as the variant character.

In this process, the items in related dictionaries should be mainly referred, but it must avoid the mechanical comparison of the number and the interpretation of the terms, and determine the relevant items firstly, collate the exegetics meaning, the borrow meaning and the false meaning, distinguish between the original intention and the actual meaning especially, then determine by the items being induced and deleted already on this basis. On the investigation of the pronunciation of the Character, it would need to restore the pronunciation of variant character to their respective generation of the historical era and the specific region, and to exclude false pronunciation and commutative pronunciation. It is mainly based on the principle of unity of glyph pronunciation and meaning of Chinese Character and draws support from the law of writing variation of glyphs has been summarized to determine the CAW.

(3) It is recommended to use the methods which Mr. Zhang Qiong summarized to identify and distinguish those character, such as the phonetic loan character, the transferred written character, the vulgar character, the private inventing character, the wrongly written character, the character invented for the proper nouns and the monomorphemic words, etc. in the specific operation.

For the phonetic loan character, it can be directly excluded if it is the temporary borrowing or the borrow meaning and the original meaning have used in parallel for a long time; if it has borrowed for a long time and the original meaning has abandoned, we should determine the relationship between them according the actual word function and the actual use in the literature, and should not identify with the other characters recording the original meaning. The transferred written character can be identified as the variant character. The vulgar character, the private inventing character and the wrongly written character can also be recognized if it is not borrowed from other character. For the character invented for the proper nouns and the monomorphemic words, it can be directly excluded 
if it is borrowed from other character, while the special inventing character can be recognized.

(4) Investigate whether the function of characters in the character set to be analyzed has differentiated during use from the functional perspective. If undifferentiated, it can be directly recognized, otherwise it should be carefully considered. There are two main types of common functional differentiation: the glyph is borrowed by other words and the extended meaning has produced (sometimes the new glyph will be invented for it). It still need to use the meaning collected by the ancient dictionaries and the actual use cases in specific analysis process. The method is the same as above.

(5) After the analysis from the configuration, it is still not appropriate to directly deny the possibility of variant relationship produced during the actual use if the character set to be analyzed is not invent for the same word. Thus, it also need to investigate whether it can be associated by homophone borrowing from the function. To respect the reality, those characters borrowed by other words for a long time and the original meaning has long been abandoned should determine with the other characters invented for the word. The variant determined through this step is called "that wasn't variant before and it's variant now".

\section{Acknowledgement}

This research was financially supported by the Youth Program of the National Social Science Fund of China (Grant No.14CYY060), the Youth Program of the Humanities and Social Science Fund of Ministry of Education of China (Grant No. 13YJCZH117), the Major Program of the National Social Science Fund of China (Grant No. 14ZDB099), the Major Program of the National Social Science Fund of China (Grant No. 15ZDB104), the Youth Program of the National Social Science Fund of China (Grant No. 16XYY015), the Youth Program of the Humanities and Social Science Fund of Ministry of Education of China (Grant No. 15XJC740004)

\section{References}

[1] Ming-Zhi YOU.On the Variant Character in Zhuanli Wanxiang Mingyi.Doctoral Dissertation of Beijing Normal University,2000:22-27.

[2] Guo-Ying LI. Definition and Types of Variant Character, Journal of Beijing Normal University (Social Sciences). 2007,(3):46-50.

[3] Jian-Wei SUN.On the Differences between Characters with Alternative Writings and Those with Alternative Configurations, Journal of Jiangsu University(Social Science Edition), 2016(2):88-92.

[4] Jing-Lun Ma.The General Theory of Chinese.Nanjing:Jiangsu Ancient Books Press, 2002:84.

[5] Qiong ZHANG. Collating of the Variant Character Corresponding to the Common Modern Chinese Character.Chengdu:Bashu Publishing House,2004:69. 